\title{
Comparison between Local Ensemble Transform Kalman Filter and PSAS in the NASA finite volume GCM - perfect model experiments
}

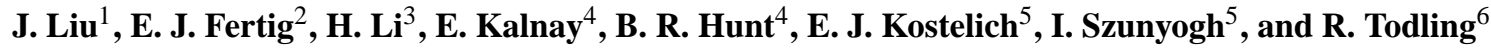 \\ ${ }^{1}$ University of California, Berkeley, CA, USA \\ ${ }^{2}$ Johns Hopkins University, Baltimore, MD, USA \\ ${ }^{3}$ Shanghai Typhoon Institute, Shanghai, China \\ ${ }^{4}$ University of Maryland, College Park, MD, USA \\ ${ }^{5}$ Arizona State University, AZ, USA \\ ${ }^{6}$ NASA-GSFC Global Modeling and Assimilation Office, Greenbelt, MD, USA
}

Received: 20 December 2007 - Revised: 4 April 2008 - Accepted: 10 July 2008 - Published: 5 August 2008

\begin{abstract}
This paper compares the performance of the Local Ensemble Transform Kalman Filter (LETKF) with the Physical-Space Statistical Analysis System (PSAS) under a perfect model scenario. PSAS is a 3D-Var assimilation system used operationally in the Goddard Earth Observing System Data Assimilation System (GEOS-4 DAS). The comparison is carried out using simulated winds and geopotential height observations and the finite volume Global Circulation Model with 72 grid points zonally, 46 grid points meridionally and 55 vertical levels. With forty ensemble members, the LETKF obtains analyses and forecasts with significantly lower RMS errors than those from PSAS, especially over the Southern Hemisphere and oceans. This observed advantage of the LETKF over PSAS is due to the ability of the 40member ensemble LETKF to capture flow-dependent errors and thus create a good estimate of the evolving background uncertainty. An initial decrease of the forecast errors in the Northern Hemisphere observed in the PSAS but not in the LETKF suggests that the LETKF analysis is more balanced.
\end{abstract}

\section{Introduction}

Three-dimensional variational data assimilation (3D-Var) was adopted for the first time in operational data assimilation at the National Centers for Environmental Prediction (NCEP) with the Spectral Statistical Interpolation (SSI) scheme in 1991 (Parrish and Derber, 1992), and has been proven to be considerably more accurate than the scheme it replaced (Optimal Interpolation, OI). Physical-Space Statistical Analysis

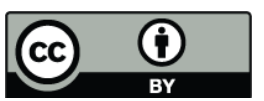

Correspondence to: J. Liu (jjliu@atmos.berkeley.edu)
System (PSAS), a 3D-Var scheme developed at NASA ${ }^{1}$, differs from other 3D-Var schemes, such as the NCEP SSI and the 3D-Var scheme of European Centre for Medium-Range Weather Forecasts (ECMWF) (Courtier et al., 1998), mainly in that it is formulated directly in physical space, rather than in spectral space (Cohn et al., 1998). It was the operational data assimilation system in the Goddard Earth Observing System Data Assimilation System (GEOS-4 DAS) (Bloom et al., 2005).

In PSAS, like in any other 3D-Var scheme, a constant statistical estimate of the background error covariance is used to represent the background uncertainty. The use of a constant background error covariance makes it difficult to adjust the background (6-h forecast) toward to the true state when there are larger than average background errors that are not well represented by the background error covariance. By contrast, ensemble Kalman filter (EnKF) schemes (Evensen, 1994; Anderson, 2001; Houtekamer and Mitchell, 2001; Bishop et

\footnotetext{
${ }^{1}$ At the time of this writing, PSAS supports the following operations at NASA: MODIS land team; Aura/MLS; Aura/TES; Aura/HIRDLS; (http://gmao.gsfc.nasa.gov/operations/candp/). GEOS-Chem group at Harvard University (e.g., http: //coco.atmos.washington.edu/cgi-bin/ion-p?page=geos_nrt.ion); FlashFLUX project team at NASA Langley Research Center (e.g., http://ams.confex.com/ams/pdfpapers/113479.pdf); Power project at NASA Langley Research Center (e.g., http://ieeexplore.ieee.org/iel5/4087812/4087813/04087926.pdf); SRB project at NASA Langley Research Center (e.g., http://eosweb.larc.nasa.gov/PRODOCS/srb/table_srb.html). It is also the operational data assimilation system at CPTEC Brazil (e.g., http://mtc-m15.sid.inpe.br/col/cptec.inpe.br/walmeida/2004/ 08.13.16.19/doc/Herdies_Data\%20assimilation.pdf), and the NWP center in Rome, Italy (e.g., http://www.wmo.ch/pages/prog/www/ DPFS/ProgressReports/2005/Italy.pdf).
} 
al., 2001; Whitaker and Hamill, 2002; Tippett et al., 2003; Ott et al., 2004; Hunt et al., 2007, Kalnay et al., 2007) estimate a flow dependent background error covariance from a time-dependent forecast ensemble. The time changing background error covariance should represent the "errors of the day" if enough ensemble members are used and the model bias is small. Recently, EnKF schemes have been shown to be able to assimilate real observations effectively. For example, Houtekamer et al. (2005) found the performance of an EnKF scheme to be comparable to the operational 3D-Var scheme when assimilating real observations into the CMC GEM grid model. With more recent changes, the performance became comparable to that of the operational 4D-Var (Houtekamer, personal communication, 2006). Whitaker et al. (2004) obtained a better mid-troposphere reanalysis from surface pressure observations with Ensemble Square Root Filter (EnSRF) (Whitaker and Hamill, 2002) than with the NCEP SSI on the GFS model at T62L28 resolution. With the same model, assimilating all operational observations except radiances, EnKF outperformed the operational SSI (Whitaker et al., 2007 and Szunyogh et al., 2008). These results show promising potential for the ensemble Kalman filter.

Unlike other ensemble Kalman filter schemes that assimilate observations serially (Anderson, 2001; Whitaker and Hamill, 2002; Houtekamer and Mitchell, 2001), the Local Ensemble Transform Kalman Filter (LETKF; Hunt et al., 2007) updates the local state variables of each grid point independently by assimilating all observations that may affect the state at that grid point. The observations are typically selected within a small local volume centered at that grid point. The localization approach of the LETKF, which is based on the localization approach of the Local Ensemble Kalman Filter (LEKF; Ott et al., 2004), makes this assimilation scheme highly parallel (Szunyogh et al., 2005, 2007). Though adapted from the LEKF, the computational cost of the LETKF is significantly lower because it solves the analysis equations in the subspace spanned by the ensemble members without using singular value decomposition. This computational efficiency, simplicity of implementation (e.g., it does not require the adjoint of the observational operator and the adjoint of the model dynamics) and its accuracy make the LETKF a particularly appealing ensemble Kalman filter scheme.

While assimilation studies based on real observations provide more realistic quantitative estimates of the accuracy of the proposed new schemes (e.g. Houtekamer et al., 2005; Whitaker et al., 2007; Szunyogh et al., 2008), studies based on simulated observations represent an important step toward better understanding the potential advantages and limitations of newly proposed schemes (e.g., Szunyogh et al, 2005). In the present study, we compare a once operational 3D-Var system (PSAS) with an EnKF (LETKF) by assimilating simulated rawinsondes on the NASA GEOS4 finite volume General Circulation Model (fvGCM, Lin, 2004). We examine and explain the differences in the performance of these two schemes and address the question of the ensemble size required to obtain an accurate background error covariance in the LETKF.

The paper is organized as follows: The LETKF and PSAS assimilation schemes are briefly described in Sect. 2. The fvGCM is described in Sect. 3. The experimental setup is explained in Sect. 4. Results comparing PSAS and the LETKF are shown in Sect. 5. Section 6 is a brief discussion of the origin of the differences in the performance of the LETKF and PSAS. Section 7 illustrates the number of ensemble members required to obtain an accurate estimate of the error covariance in the LETKF scheme. Section 8 is a summary and discussion.

\section{Assimilation schemes}

\subsection{Physical-Space Statistical Analysis System (PSAS)}

PSAS (Cohn et al., 1998) solves the standard analysis equations (Eqs. 1 and 2) to minimize the analysis error variance

$\boldsymbol{x}^{a}=\boldsymbol{x}^{b}+\mathbf{K}\left[\boldsymbol{y}^{o}-h\left(\boldsymbol{x}^{b}\right)\right]$

$\mathbf{K}=\mathbf{P}^{b} \mathbf{H}^{T}\left(\mathbf{H} \mathbf{P}^{b} \mathbf{H}^{T}+\mathbf{R}\right)^{-1}$

Here, $h(\bullet)$ is a nonlinear observation operator transforming the model state variables into observation space, $\mathbf{H}$ is its linearized (Jacobian) operator and $\mathbf{H}^{T}$ is the transpose (adjoint) of the Jacobian.

Unlike other 3D-Var schemes, PSAS performs most of its computations in the space of observations (Cohn et al., 1998). More specifically, PSAS applies a conjugate gradient (CG) algorithm to obtain an intermediate variable $\boldsymbol{w}$ from the following equation:

$\left(\mathbf{H} \mathbf{P}^{b} \mathbf{H}^{T}+\mathbf{R}\right) \boldsymbol{w}=\boldsymbol{y}^{o}-h\left(\boldsymbol{x}^{b}\right)$

$\boldsymbol{w}$ is then substituted into Eqs. (1) and (2) to obtain the updated analysis state

$\boldsymbol{x}^{a}=\boldsymbol{x}^{b}+\mathbf{P}^{b} \mathbf{H}^{T} \boldsymbol{w}$

Together, Eqs. (3) and (4) are referred to as the PSAS equations. In the specification of the error covariance, only the matrices $\mathbf{H} \mathbf{P}^{b} \mathbf{H}^{T}+\mathbf{R}$ and $\mathbf{P}^{b} \mathbf{H}^{\mathbf{T}}$ are calculated and stored. These matrices depend on the observation types.

The most computationally expensive part in PSAS is to solve Eq. (3), which depends on the number of observations. Therefore, the computational cost of PSAS depends primarily on the number of observations, not on the number of degrees of freedom in the model. It is more efficient to use PSAS than the other 3D-Var systems to assimilate rawinsondes, since there are fewer rawinsonde observations than the number of degrees of freedom in the dynamical model. Because of this efficiency, as well as the availability of the PSAS code for installation on our computer system (a 25 
dual processor PC cluster with $2.8 \mathrm{GHz}$ dual Xeon speed), we chose PSAS as the standard 3D-Var assimilation scheme with which to compare the LETKF. Also, the version of PSAS available for this study was developed to assimilate geopotential height observations, so we also assimilate geopotential height observations in the comparison of PSAS with the LETKF.

\subsection{The Local Ensemble Transform Kalman Filter}

A detailed description of the LETKF is given in Hunt et al. (2007). In the following sub-sections, we briefly summarize the algorithm and its application on the fvGCM. For this application, we first determine the ensemble forecasts and the ensemble forecasts at observation locations (Sect. 2.2.1), then do localization around each grid point (Sect. 2.2.2). Each central grid point is updated in parallel using local information (Sect. 2.2.3), and finally the analysis ensemble at every grid point are combined to obtain a global analysis for each ensemble member (Sect. 2.2.4).

\subsubsection{Global ensemble forecasts}

First, an ensemble of $k$ forecasts, the $i$ th of which is denoted by $\boldsymbol{x}_{g}^{b(i)}$, are created by integrating the fvGCM from each analysis ensemble member valid at the previous analysis time. Then, each of the forecasts is transformed into observation space by applying the observation operator. The output is denoted as $\boldsymbol{y}_{g}^{b(i)}=h\left(\boldsymbol{x}_{g}^{b(i)}\right)$, where the sub-index $g$ represents global vectors.

\subsubsection{Localization and parallelization}

A distinguishing characteristic of the LETKF is its localization scheme. Most ensemble Kalman filter techniques introduce covariance localization (Houtekamer and Mitchell, 2001; Hamill et al., 2001) to avoid the spurious long-distance correlations introduced by sampling with a limited number of ensemble members. The version of the LETKF used in this paper addresses this problem by cutting off a local region around each grid point (Ott et. al., 2004; Hunt et al., 2007). Only the information within the local box is used to update the center point ${ }^{2}$. There is substantial overlap between adjacent local regions corresponding to the neighboring grid points (Szunyogh et al., 2005). The great overlap between adjacent local boxes implies the overlap of the observation data set assimilated at the adjacent grid points, which ensures spatial continuity of the analysis (Ott et al., 2004).

\footnotetext{
${ }^{2}$ Alternatively, the localization can be based on the choice of the observations used at each grid point (Hunt et al., 2007). This approach has some advantages over the box localization adopted here, especially near the poles and for satellite observations that may depend on the model state in a less local way than the conventional observations.
}

Because the state is updated independently at each grid point, the wall-clock time needed to finish a LETKF analysis can be dramatically reduced by parallel computation. For our application, the parallel implementation is realized by separating the whole globe into a number of latitude strips based on the number of available processors. The analysis of each latitude strip is computed independently on different processors. The final analysis results do not depend on the available processors, because the assimilated observations at each local box are independent of the number of processors, and only depend on the localization scale.

Due to localization, all the vectors presented in the next subsection are reduced from global to local size. In this way, rather than having to assimilate observations serially (one after the other) as in several other ensemble Kalman filter techniques (Tippett et al., 2003), the LETKF assimilates all relevant observations simultaneously (Ott et al., 2004; Szunyogh et al., 2005). Simultaneous assimilation, which allows for observation error correlations in space, is particularly important when the observation coverage is dense and correlated, such as for satellite observations. It can also assimilate observations at the appropriate time when the 4-D-LETKF extension is used, which allows for observation error correlations in time as well (Hunt et al., 2004; Hunt et al., 2007; Kalnay et al., 2007).

\subsubsection{Local analysis}

Within each latitude strip, the analysis of each grid point is performed sequentially. As described by Szunyogh et al. (2005), at each grid point, the local background vector $\boldsymbol{x}^{b(i)}$, the corresponding local background vector in observation space $\boldsymbol{y}^{b(i)}$, and the local observation vector $\boldsymbol{y}^{o}$ only include the information within the local box. Different localizations may be chosen for different observations, such as different localization for satellite radiances (Fertig et al., 2007). Within the local box, the background state is defined as the ensemble mean of the local forecast vectors:

$\overline{\boldsymbol{x}}^{b}=k^{-1} \sum_{i=1}^{i=k} \boldsymbol{x}^{b}(i)$

Unlike the LEKF (Ott et. al., 2004; Szunyogh et al., 2005), the LETKF does not calculate the background error covariance explicitly. It calculates the background ensemble perturbations $\mathbf{X}^{b}$ instead. Its $i$ th column is given by $\boldsymbol{x}^{b(i)}-\overline{\boldsymbol{x}}^{b}$. Following Hunt et al. (2007), the ensemble perturbations in this local box are given by

$\mathbf{X}^{a}=\mathbf{X}^{b}\left(\mathbf{P}^{a}\right)^{\frac{1}{2}}$,

It is the transformation of the background ensemble perturbations by $\mathbf{P}^{a 1 / 2}$, where $\mathbf{P}^{a}$, the analysis error covariance in the ensemble space, is given by

$$
\mathbf{P}^{a}=\left[(k-1) \mathbf{I}+\mathbf{Y}^{b T} \mathbf{R}^{-1} \mathbf{Y}^{b}\right]^{-1} .
$$


Table 1. Observation error standard deviations as a function of vertical level for simulated zonal wind (U), meridional wind (V) and geopotential height $(\mathrm{H})$ observations (adapted from PSAS).

\begin{tabular}{lccl}
\hline Unit $(\mathrm{hPa})$ & $\mathrm{U}(\mathrm{m} / \mathrm{s})$ & $\mathrm{V}(\mathrm{m} / \mathrm{s})$ & $\mathrm{H}(\mathrm{m})$ \\
\hline 1000 & 2.0 & 2.0 & 5.4 \\
850 & 2.2 & 2.2 & 5.6 \\
700 & 2.3 & 2.3 & 6.2 \\
500 & 2.7 & 2.7 & 8.6 \\
400 & 3.2 & 3.2 & 10.8 \\
300 & 3.4 & 3.4 & 12.8 \\
250 & 3.4 & 3.4 & 13.5 \\
200 & 3.3 & 3.3 & 14.5 \\
150 & 2.7 & 2.7 & 16.3 \\
100 & 2.7 & 2.7 & 19.3 \\
\hline
\end{tabular}

$\mathbf{Y}^{b}$ is the matrix of ensemble background perturbations in observation space with the $i$ th column given by $\boldsymbol{y}^{b(i)}-\overline{\boldsymbol{y}}^{b}$, where $\overline{\boldsymbol{y}}^{b}=k^{-1} \sum_{i=1}^{i=k} \boldsymbol{y}^{b(i)}$. Because $\mathbf{Y}^{b}$ is formulated using the nonlinear observation operator, unlike 3D-Var or 4D-Var methods, the LETKF does not require either the Jacobian $\mathbf{H}$ of the observation operator or its adjoint $\mathbf{H}^{T}$. The ensemble mean state of LETKF is updated by the equation:

$\overline{\boldsymbol{x}}^{a}=\overline{\boldsymbol{x}}^{b}+\mathbf{X}^{b} \tilde{\mathbf{P}}^{a} \mathbf{Y}^{b T} \mathbf{R}^{-1}\left(\boldsymbol{y}^{o}-\overline{\boldsymbol{y}}^{b}\right)$

The analysis ensemble is given by adding the analysis mean to the analysis perturbations: $\boldsymbol{x}^{a(i)}=\mathbf{X}^{a(i)}+\overline{\boldsymbol{x}}^{a}$.

\subsubsection{Global analysis ensemble}

The local analysis described above returns the analysis ensemble for the center grid point of the local box. The analysis for each grid point in the latitude strip is then connected to return a single file for each strip including all ensemble members. The global analysis for each ensemble member is then extracted from these files. The global analysis ensemble is then used to initiate the ensemble forecasts discussed in Sect. 2.2.1.

\section{NASA fvGCM}

The dynamical core of the NASA GEOS 4 is the fvGCM developed by Lin (2004) with highly accurate numerical discretization. The fvGCM solves the governing equations by employing a Lagrangian vertical coordinate. Unlike many models that forecast surface pressure, the NASA fvGCM forecasts the pressure thickness $(\delta p)$ between vertical model levels and updates surface pressure $(P s)$ as a diagnostic variable. The fvGCM also forecasts zonal wind $(u)$, meridional wind $(v)$, scaled potential temperature $(\theta)$, and specific humidity $(q)$.
The version of the fvGCM employed in our experiments has a horizontal resolution of $5^{\circ}$ longitude and $4^{\circ}$ latitude (72 zonal and 46 meridional grid points). The model has 55 vertical levels and includes a very high top at $0.01 \mathrm{hPa}$. We note that the horizontal resolution is coarser than that used operationally, but this allows performing a large number of experiments under our limited computational resources.

\section{Simulated observations and experimental design}

The assimilation experiments described in this study were conducted in the perfect model scenario. A nature run, representing the true state of the atmosphere, was created by running the NASA fvGCM for three months from the operational analysis of 16 December 2002. Simulated rawinsonde observations were obtained by converting the true model state to rawinsonde variable types, interpolating this converted true state to the real rawinsonde locations, and then adding zero-mean non-correlated Gaussian distributed noise with standard deviations same as the operationally assumed rawinsonde errors (Table 1). The observations are at the real rawinsonde observation locations shown in the left panel of Fig. 2 for 00:00 UTC, including zonal wind, meridional wind and geopotential height. A similar number of observations are available at 12:00 UTC, but far fewer are available at 06:00 UTC and 18:00 UTC (not shown).

The initial analysis cycle started at 18:00 UTC on 16 December 2002 for PSAS. The initial condition used for this PSAS run was the true state from the nature run at 00:00 UTC on 15 January 2003. The LETKF analysis cycle was started on 18:00 UTC on 01 January 2003 and used the PSAS analysis as the initial mean state of the ensemble. The initial ensemble members were obtained by adding normally distributed noise to the mean analysis state. The standard deviation of the analysis ensemble perturbations was the same as the standard deviation of the observational noise for observed variables and $0.25 \mathrm{~K}$ for scaled potential temperature.

The PSAS obtains analysis increments of the observed variables, and then converts these increments to update the model state variables (see Eqs. 3 and 4). The LETKF, on the other hand, directly obtains analyses for the model variables. In this study, the LETKF directly updates zonal and meridional wind, scaled potential temperature, and surface pressure. Surface pressure is not a prognostic variable, but it is used to update the related prognostic variable, pressure thickness. For simplicity and efficiency in this study, pressure thickness is updated proportionally to the surface pressure increment for each ensemble member. Specifically, the analysis increment of the pressure thickness at level $k$ for the $i$ th ensemble member is given by

$$
\frac{\Delta \delta p_{k}^{a(i)}}{\delta p_{k}^{b(i)}}=\frac{\Delta P s_{k}^{a(i)}}{P s_{k}^{b(i)}},
$$




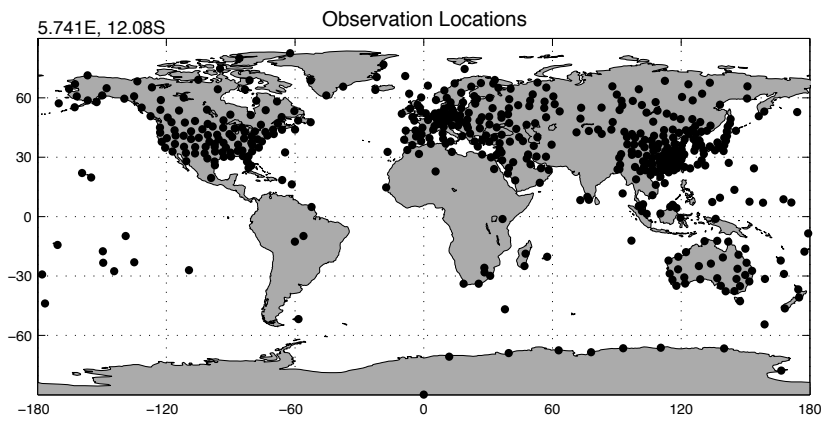

Fig. 1. Left panel: real rawinsonde observation locations (black dots) at 00:00 UTC. Right panel: relative distribution of observational coverage at different pressure levels.

where $\Delta$ indicates the analysis increment of the corresponding variable. Furthermore, neither the LETKF nor the PSAS updates specific humidity in this study to avoid the complexities of assimilating humidity observations (Dee and DaSilva, 2003). We have a separate study examining the impact of assimilating humidity with the LETKF (Liu, 2007).

Sampling errors and the effects of nonlinearities in the evolution of the estimation errors can lead to an underestimation of the background error covariance and to filter divergence (e.g., Whitaker and Hamill, 2002). To compensate for this problem, a multiplicative variance inflation scheme (Anderson and Anderson, 1999) is employed in the LETKF. In practice, we achieve this as in Hunt et al. (2007) by modifying Eq. (7):

$\tilde{\mathbf{P}}^{a}=\left[\frac{(k-1)}{1+\rho} \mathbf{I}+\mathbf{Y}^{b T} \mathbf{R}^{-1} \mathbf{Y}^{b}\right]^{-1}$,

where $\rho$ is a positive number, and $(1+\rho)$ is the inflation factor. The main difference between the approach of Anderson and Anderson (1999) and that of Hunt et al. (2007) is that while the former scheme inflates the ensemble background perturbations, Eq. (10) inflates the analysis perturbations. These two approaches would be equivalent if the evolution of the ensemble perturbations is strictly linear. The inflation factor, $\rho$, is tuned to change with vertical levels, latitudes, and time (see Tables 2 and 3). At the lower levels the inflation factor was kept constant throughout the assimilation cycle ( $8 \%$ over the entire globe). For the levels above $100 \mathrm{hPa}$, the inflation factor was larger during spin-up time. We found experimentally that such large inflation above $100 \mathrm{hPa}$ (Table 2) is useful for the first few analysis steps before the analysis error settles at a stationary level. Once the system settles, the inflation is decreased from $8 \%$ at $100 \mathrm{hPa}$ to about $5 \%$ over the polar region (Table 3). To avoid changing inflation factor abruptly either in vertical or meridional direction, we linearly change these inflation factors. In the local boxes where there is no observation, we do not inflate the background, though later studies have found that the analysis improves slightly
Table 2. Inflation factors as a function of vertical levels and latitude bands for spin-up.

\begin{tabular}{lcccc}
\hline Unit: hPa & $90^{\circ} \mathrm{S}-14^{\circ} \mathrm{S}$ & $10^{\circ} \mathrm{S}-6^{\circ} \mathrm{N}$ & $10^{\circ} \mathrm{N}-26^{\circ} \mathrm{N}$ & $30^{\circ} \mathrm{N}-90^{\circ} \mathrm{N}$ \\
\hline $976.7-118.2$ & 1.08 & 1.08 & 1.08 & 1.08 \\
100.5 & 1.09 & 1.09 & 1.09 & 1.09 \\
85.4 & 1.10 & 1.10 & 1.10 & 1.10 \\
72.6 & 1.20 & 1.20 & 1.20 & 1.20 \\
61.5 & 1.25 & 1.30 & 1.30 & 1.30 \\
52.0 & 1.35 & 1.40 & 1.40 & 1.40 \\
43.9 & 1.35 & 1.50 & 1.50 & 1.50 \\
37.0 & 1.35 & 1.50 & 1.60 & 1.60 \\
31.1 & 1.35 & 1.50 & 1.70 & 1.70 \\
26.0 & 1.35 & 1.50 & 1.70 & 1.80 \\
21.8 & 1.35 & 1.50 & 1.70 & 1.90 \\
$18.1-0.015$ & 1.35 & 1.50 & 1.70 & 2.00 \\
\hline
\end{tabular}

by also inflating the background in these regions (Szunyogh et al., 2007).

The dimensions of the local box were varied spatially to account for inhomogeneous observation coverage and the change of physical distance between grid points with latitudes. The width of the box was increased in the Southern Hemisphere ( $\mathrm{SH})$, where observations are sparse. To account for the convergence of the meridians toward the poles, the number of grid points in zonal direction included in the local box was also increased with latitude in both Hemispheres. For example, the horizontal local patch was 7 grid points by 7 grid points in the mid-latitudes in the Northern Hemisphere $(\mathrm{NH})$, while it was increased to 15 grid points by 7 grid points near the poles. The vertical dimension of the local boxes contained 3 vertical levels, except at the top and the bottom model levels where they contained 1 level. In the newer configuration of the LETKF (Hunt et al., 2007), the localization scale only depends on the actual physical distance, requiring less tuning.

We tuned the variance of background error covariance used by PSAS to account for the fact that it was originally tuned using real observations and found that the results were not very sensitive to this amplitude. Since we did not tune the correlation structure, the PSAS results presented below may not be optimal. So the advantage of the LETKF over PSAS may not be as large as the results shown here.

\section{Relative performance of the LETKF and the PSAS}

We evaluate the performance of both PSAS and the LETKF by computing the Root Mean Square (RMS) errors for both the analyses and the forecasts. The relative accuracy of these two schemes is examined by comparing the magnitude of the analysis RMS errors, which includes the $500 \mathrm{hPa}$ RMS error time series averaged over the globe, the global and time average RMS errors over all vertical levels, and the zonal mean RMS error. We also calculate the percentage improvement of 
Table 3. Inflation factors as a function of vertical levels and latitude bands for stable state.

\begin{tabular}{lcccccc}
\hline Unit: hPa & $90^{\circ} \mathrm{S}-62^{\circ} \mathrm{S}$ & $90^{\circ} \mathrm{S}-14^{\circ} \mathrm{S}$ & $10^{\circ} \mathrm{S}-6^{\circ} \mathrm{N}$ & $10^{\circ} \mathrm{N}-50^{\circ} \mathrm{N}$ & $54^{\circ} \mathrm{N}-66^{\circ} \mathrm{N}$ & $70^{\circ} \mathrm{N}-90^{\circ} \mathrm{N}$ \\
\hline $976.7-18.2$ & 1.08 & 1.08 & 1.08 & 1.08 & 1.08 & 1.08 \\
100.5 & 1.09 & 1.09 & 1.09 & 1.09 & 1.09 & 1.09 \\
85.4 & 1.10 & 1.10 & 1.10 & 1.10 & 1.10 & 1.10 \\
72.6 & 1.09 & 1.15 & 1.20 & 1.20 & 1.20 & 1.10 \\
61.5 & 1.08 & 1.20 & 1.30 & 1.30 & 1.30 & 1.10 \\
52.0 & 1.07 & 1.20 & 1.30 & 1.40 & 1.30 & 1.10 \\
43.9 & 1.06 & 1.20 & 1.30 & 1.50 & 1.30 & 1.10 \\
37.0 & 1.05 & 1.20 & 1.30 & 1.60 & 1.30 & 1.10 \\
31.1 & 1.05 & 1.20 & 1.30 & 1.60 & 1.30 & 1.10 \\
26.0 & 1.05 & 1.20 & 1.30 & 1.60 & 1.30 & 1.10 \\
21.8 & 1.05 & 1.20 & 1.30 & 1.60 & 1.30 & 1.10 \\
$18.1-0.015$ & 1.05 & 1.20 & 1.30 & 1.60 & 1.30 & 1.10 \\
\hline
\end{tabular}
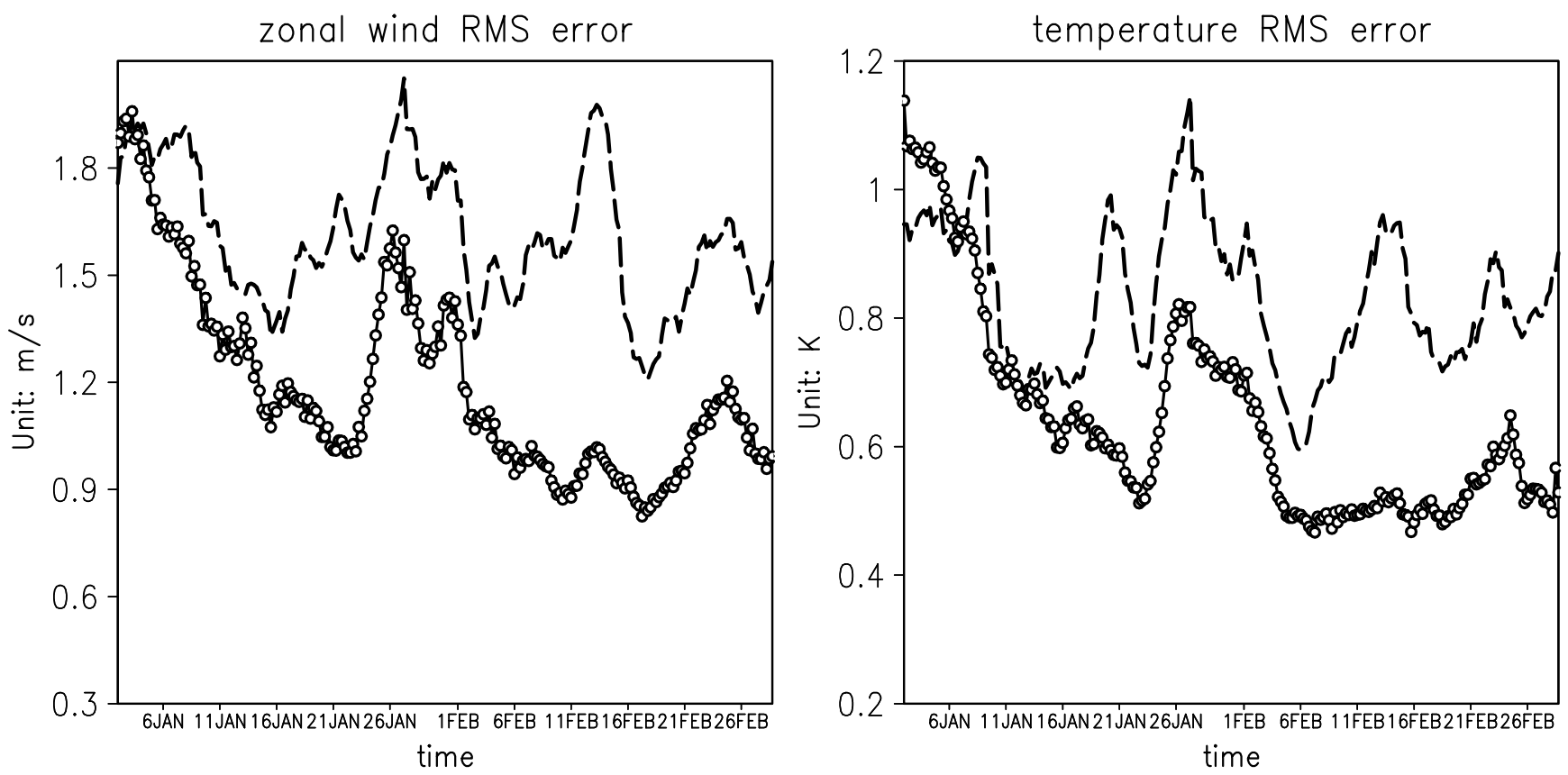

Fig. 2. $500 \mathrm{hPa}$ global average analysis RMS error (y-axis) as function of time (x-axis) for zonal wind (left panel) and temperature (right panel). Dashed line: PSAS; solid line with open circles: the LETKF.

LETKF over PSAS (RMS error difference between LETKF and PSAS normalized by the PSAS RMS error). Negative values indicate that LETKF is better than PSAS. For the forecasts, we compare the 5-day forecast RMS error from these two schemes in different areas, as well as the representation and the impact of gravity waves on the forecast.

\subsection{Time series of analysis RMS error}

The RMS error time series start from 00:00 UTC, 2 January 2003, when PSAS analysis has already settled, and the LETKF analysis cycle begins. As shown in Fig. 2, after a few days the LETKF analysis (solid line with open circles) has smaller errors than the PSAS analysis (dashed line) for both zonal wind (left panel) and temperature (right panel). After the initial transient period, the differences between the RMS errors of these two schemes are large and significant. Beyond the transient period, the RMS error of the LETKF analysis is not only smaller, but also shows less variability than that of PSAS. The difference is especially apparent on 12 February, when PSAS has a large spike in the RMS error. At this time, the RMS error of the LETKF (solid line with open circles in Fig. 2) only has slight fluctuations. We will further explore the reasons for this difference in error fluctuations in Sect. 6.

The RMS error over the $\mathrm{NH}\left(22^{\circ} \mathrm{N}-90^{\circ} \mathrm{N}\right)$ (Fig. 3) is much smaller and shows less variability than the global RMS 

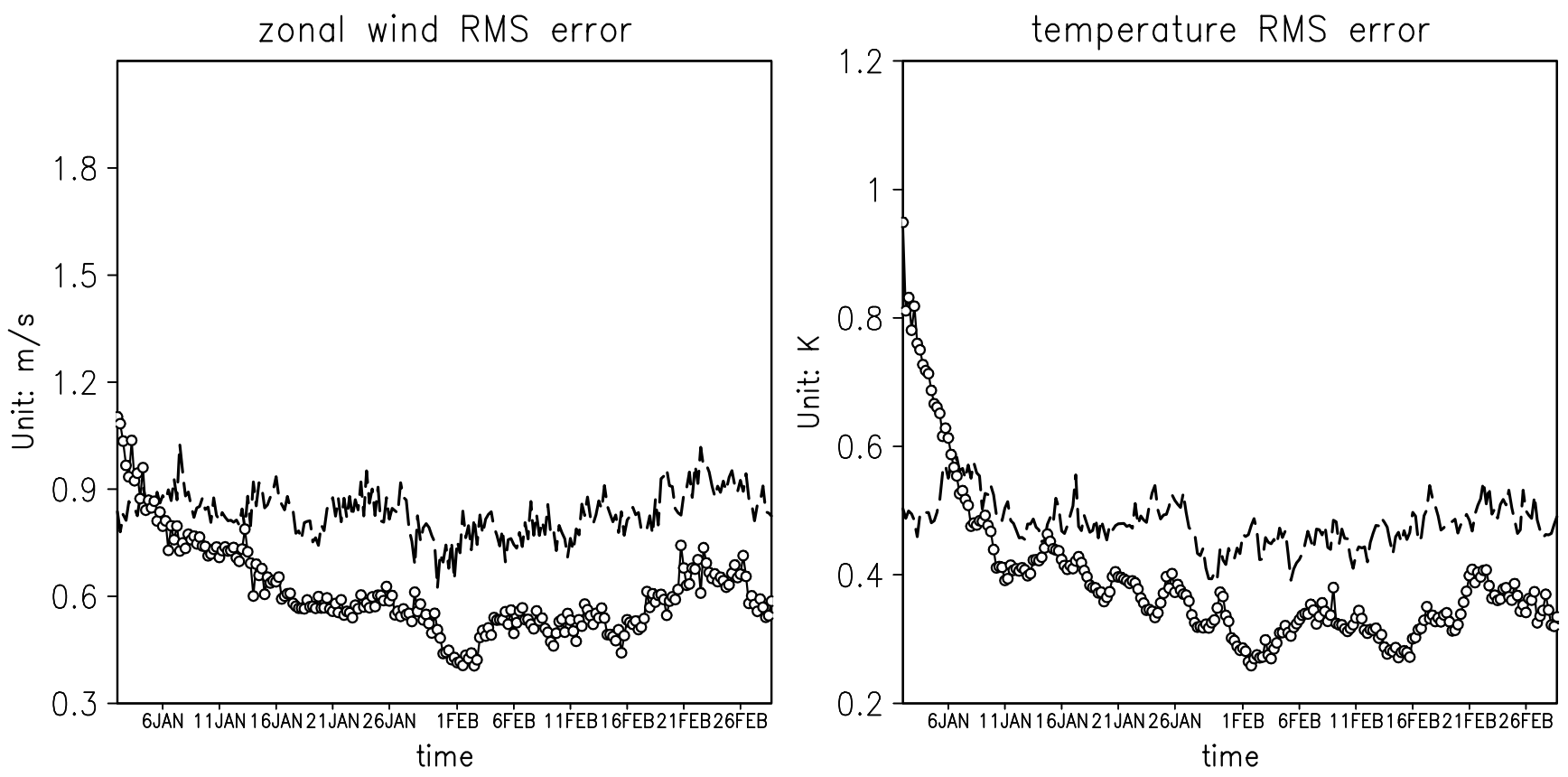

Fig. 3. Same as Fig. 2, except for the analysis RMS error averaged over the Northern Hemisphere $\left(22^{\circ} \mathrm{N}-90^{\circ} \mathrm{N}\right)$.

error for both schemes. Because the density of rawinsonde network is highest in the NH (Fig. 1), the background quickly adjusts to the observations there. Therefore, most of the fluctuations of the errors appear in the regions with low observation density, like the extratropical $\mathrm{SH}\left(22^{\circ} \mathrm{S}-90^{\circ} \mathrm{S}\right)$ and oceans.

\subsection{Vertical and latitudinal structure of the analysis error}

Figures 2 and 3 show that the LETKF analysis RMS errors at $500 \mathrm{hPa}$ are smaller than those of PSAS. We further compare the RMS errors at all vertical levels below $100 \mathrm{hPa}$, shown in Fig. 4. It shows that the LETKF analysis RMS errors are smaller at all model levels for both zonal wind (left panel) and temperature (right panel). The RMS error is significantly smaller than the observational error (Table 1) for both PSAS (dashed line in Fig. 4) and the LETKF (solid line with open circles in Fig. 4). The percentage improvement relative to PSAS is about $40 \%$ for zonal wind and $30 \%$ for temperature (not shown). The improvement is larger at the lower levels than at the higher levels. For the levels above $100 \mathrm{hPa}$ (not shown), the RMS errors of both the LETKF and PSAS increase sharply, with the LETKF increases faster. At around $80 \mathrm{hPa}$, the accuracy of PSAS surpasses the LETKF. We need to further investigate the reason for the better performance of PSAS above $100 \mathrm{hPa}$.

Figure 5 compares zonally and temporally averaged analysis RMS errors from PSAS and LETKF. In the NH, where both schemes provide more accurate analysis, the LETKF has smaller errors than PSAS (bottom left panel in Fig. 5).
The zonal wind analysis RMS error of the LETKF is only between $0.25 \mathrm{~m} / \mathrm{s}$ and $0.5 \mathrm{~m} / \mathrm{s}$ at high latitudes (top left panel in Fig. 5), which is about $15 \%$ to $25 \%$ of the observation error. In most of the tropics and $\mathrm{SH}$, where the RMS errors are larger, the difference between the RMS error of the LETKF and PSAS is also larger (bottom left panel in Fig. 5), especially over SH. Although the RMS error over the tropics is large for both schemes, the error reduction of LETKF compared with PSAS is between $-30 \%$ and $-40 \%$ in this region (bottom right panel in Fig. 5). The percentage improvement is between $-40 \%$ and $-50 \%$ through the whole vertical column over the mid-latitudes in the $\mathrm{SH}$ (bottom right panel in Fig. 5). However, the percentage improvement becomes smaller beyond $70^{\circ} \mathrm{S}$ toward the South Pole, where the LETKF analysis becomes slightly worse than PSAS. The assimilation near the poles was a challenge for the present local box formulation of LETKF (see footnote in Sect. 2.2.2).

\subsection{Comparison of forecast errors}

Since in the perfect model scenario forecast errors originate only from the errors in initial condition, and the analysis state of the LETKF has smaller errors than PSAS, better forecasts from the LETKF are expected. Also note that if the initial conditions are in balance, growing errors present in the initial conditions will grow exponentially (essentially like bred vectors generated by the analysis cycle, Toth and Kalnay, 1997). If the initial conditions are not in balance, however, the initial errors will not necessarily grow, and may even decay during the geostrophic adjustment period. Only after the unbalanced 

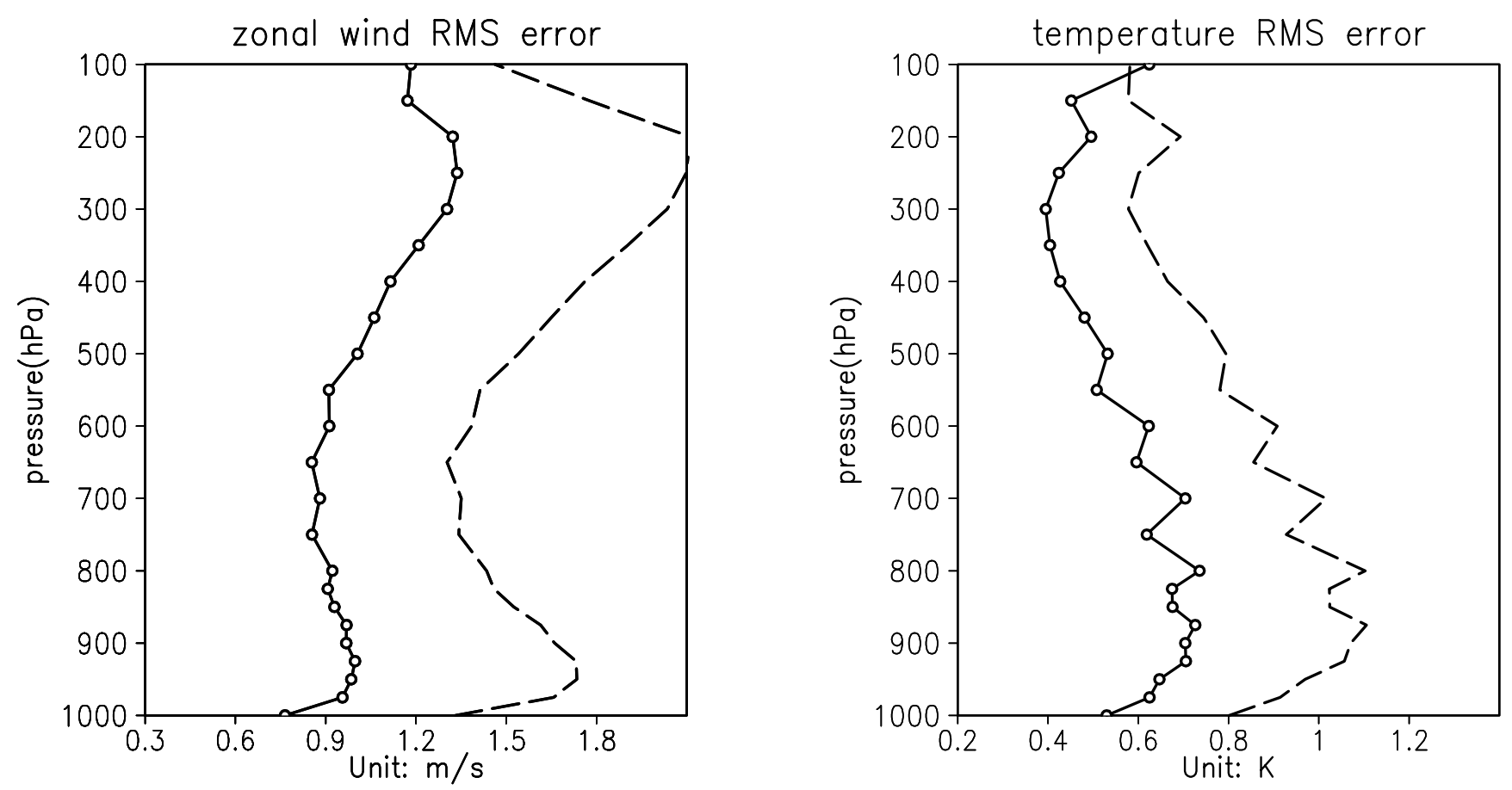

Fig. 4. Time mean (averaged over February) of the analysis RMS error averaged over the globe as function of vertical levels for zonal wind (top left panel) and temperature (top right panel). Dashed line: PSAS; solid line with open circles: the LETKF.

errors disperse, and the growing modes start dominating the error, do we expect to observe exponential growth. Therefore, the error growth observed in different areas provides an indication of the type of errors and the relative balance of the analysis.

As expected, the forecast RMS errors from the LETKF analysis are smaller than that from PSAS analysis (dashed line) during a 5-day forecast period over all the regions (Fig. 6). However, different regions show different characteristics of error growth. In the NH (top left panel in Fig. 6), PSAS errors initially decay, and start growing after about a day, indicating that the PSAS analyses are not well balanced. With the same set of observations, the LETKF starts with smaller errors, but they grow faster. In the SH (top right panel in Fig. 6), the errors of PSAS start growing immediately after the analysis, suggesting that the PSAS analysis is more balanced in the SH than in the NH. This is not surprising, since the number of observations in the $\mathrm{SH}$ is much smaller than in the $\mathrm{NH}$, and it is the assimilation of observations that cause the PSAS analysis to lose its balance (The assimilation of observations takes place within the subspace spanned by the PSAS background error covariance). The forecast RMS errors of LETKF grow at a smaller rate than PSAS after an initial slight decrease during the first $6 \mathrm{~h}$. The initial error decrease is presumably associated with the convective imbalance in the SH summer (Harlim et al., 2005). In the tropics $\left(22^{\circ} \mathrm{S}-22^{\circ} \mathrm{N}\right.$, bottom left panel in Fig. 6), the forecast RMS errors of PSAS are almost constant for a couple of days, and then increase linearly. For the LETKF the fore- cast RMS errors start smaller than PSAS and grow linearly with time at about the same rate as PSAS. This characteristic linear growth of errors in the tropics was also observed in Kuhl et al. (2007) in simulations with the NCEP Global Forecasting System (GFS). Unlike extratropical error growth dominated by slow baroclinic waves, tropical errors are dominated by convection, which saturate almost immediately at small scales and slowly propagate to larger scales even in a perfect model scenario (Kalnay, 2003; Harlim et al., 2005).

\subsection{Accuracy in representing gravity waves}

Maintaining atmospheric balance is particularly important during data assimilation. Imbalance in the atmospheric analysis will result in the excitation of spurious gravity waves, which eventually will ruin the accuracy of the data assimilation system. Gravity waves are generally not observed with significant amplitudes, except for the diurnal and semidiurnal tides. So the goal during data assimilation is to retain the gravity waves present in reality, but to avoid exciting spurious gravity waves.

Though geostrophic balance is imposed in the construction of the background error covariance, some of the other constraints are still needed in some statistical optimization problem. It is common to apply a balancing algorithm such as nonlinear normal mode initialization (e.g., Daley, 1991), digital filter (Lynch and Huang, 1992) or the Incremental Analysis Update (Bloom et al, 1996) to 3D-Var in order to eliminate high frequency waves before the next forecast 

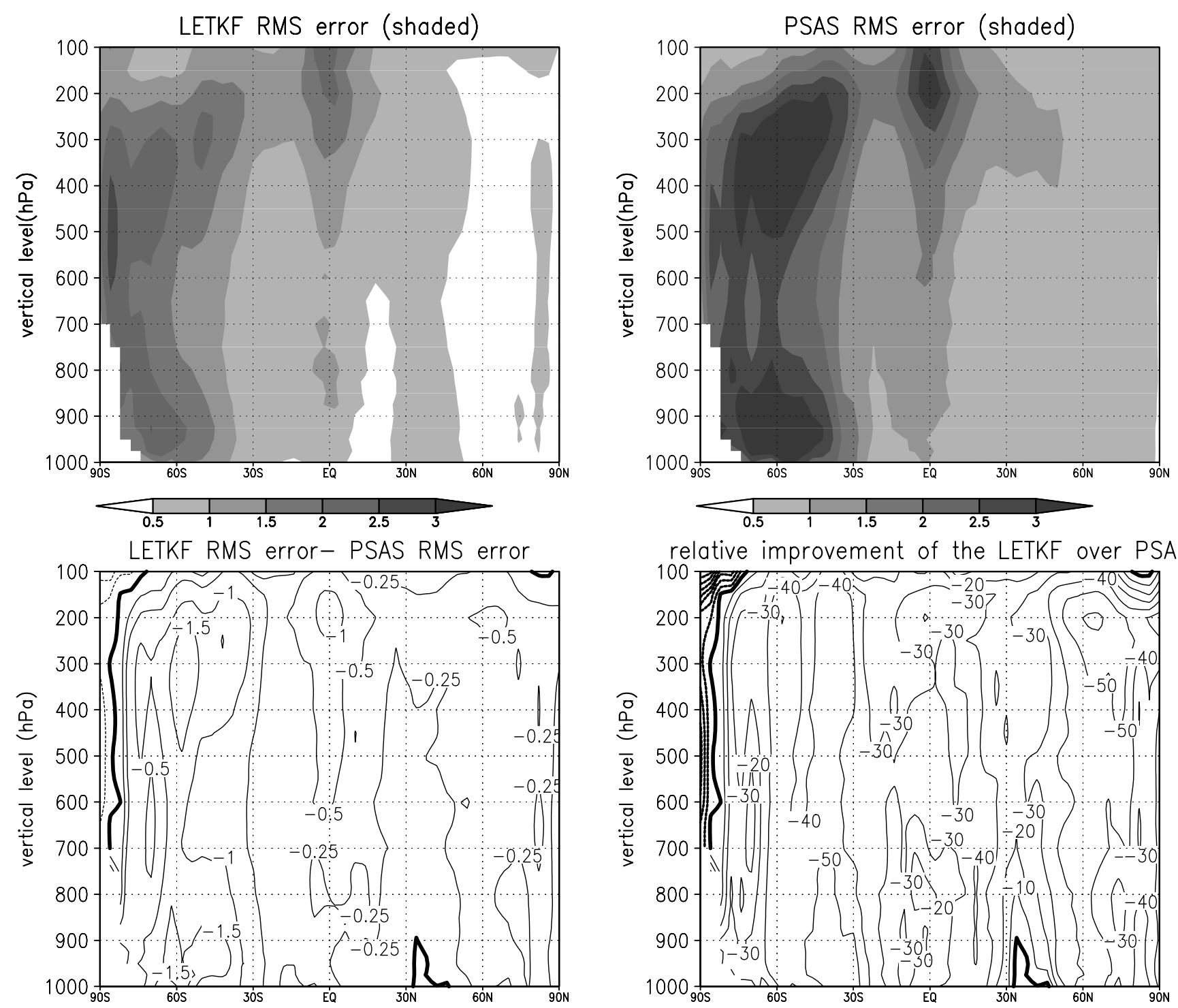

Fig. 5. The contours in the two figures in top panel indicate the time average (over February) of the true zonal wind field, and the shades indicate the zonal average of the time mean zonal wind analysis RMS error of the LETKF (top left panel) and PSAS (top right panel). Bottom left panel is the analysis RMS error difference between the LETKF and PSAS, and the bottom right panel is the relative improvement of the LETKF over PSAS. The thicker line in both figures in the bottom panel is zero line.

begins. In the current implementation of both the PSAS and the LETKF, there is no such extra constraint. The ensemble analyses in the LETKF minimize the introduction of spurious gravity waves by computing the analyses as a linear combination of the ensemble forecasts, which are generally well balanced. Fast gravity waves remain in the analysis field only if they are in the background. Although the use of local boxes in the LETKF could lead to imbalances, the large overlap between different local boxes (as discussed in Sect. 2) in the LETKF is apparently able to minimize the excitement of gravity waves by assimilating similar information in neighboring regions. We compare the relative ability of both schemes in representing real gravity waves by plotting the error difference (upper right panel in Fig. 7) between the analyzed horizontal divergence and the truth (upper left panel in Fig. 7) for a period at the location $\left(32^{\circ} \mathrm{N}, 93^{\circ} \mathrm{W}\right.$ on $700 \mathrm{hPa}$ ) where a large amplitude gravity wave is observed in the true dynamical field. The error of the divergence field from the LETKF (open circles in the right panel in Fig. 7) is smaller than that of PSAS (closed circles in Fig. 7). In agreement with the results of Szunyogh et al. (2005), we do not observe spurious high frequency gravity waves. PSAS (closed circles) also gives a fairly good analysis of the true gravity waves, but the amplitude of the errors is considerably 

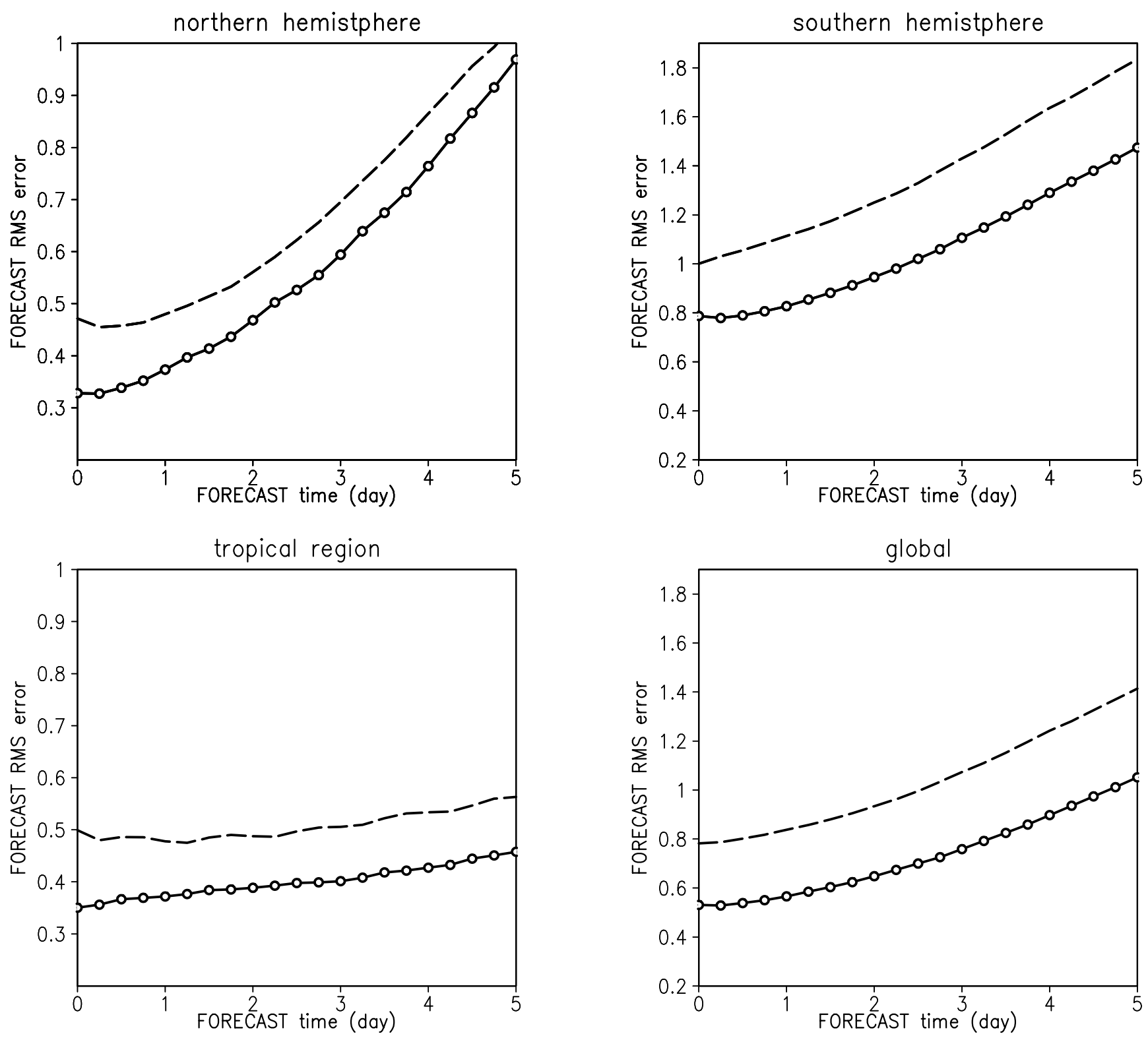

Fig. 6. $500 \mathrm{hPa}$ zonal wind forecast RMS error $(\mathrm{m} / \mathrm{s})$, averaged over February, as a function of the leading time in different regions. Top left panel: Northern Hemisphere $\left(22^{\circ} \mathrm{N}-90^{\circ} \mathrm{N}\right)$; Top right panel: Southern Hemisphere $\left(22^{\circ} \mathrm{S}-90^{\circ} \mathrm{S}\right)$; Bottom left panel: Tropics $\left(22^{\circ} \mathrm{S}-\right.$ $22^{\circ} \mathrm{N}$ ); Bottom right panel: the globe. Dashed line: PSAS; solid line with open circles: the LETKF.

larger than that of LETKF. The gravity wave that appears in the truth has both diurnal and semi-diurnal components, especially around 14 February. This structure is more apparent in the 2-day forecasts starting on 12:00 UTC 14 February plotted every hour (bottom panel in Fig. 7). The forecasted surface pressure shows the diurnal and semidiurnal modes in the truth (crosses), PSAS (full circles), and LETKF (open circles) forecasts. Although both forecasts capture the diurnal and semidiurnal tides, we observe that the initial conditions from the LETKF lead to a more balanced and accurate forecast. We have to note that the observation coverage in the current experimental setup is rather sparse and the assimilation of observations may introduce imbalance, so whether 3D-Var system and the LETKF can still maintain balance without any other constraints in case of more dense observation coverage needs further investigation in a future study. 

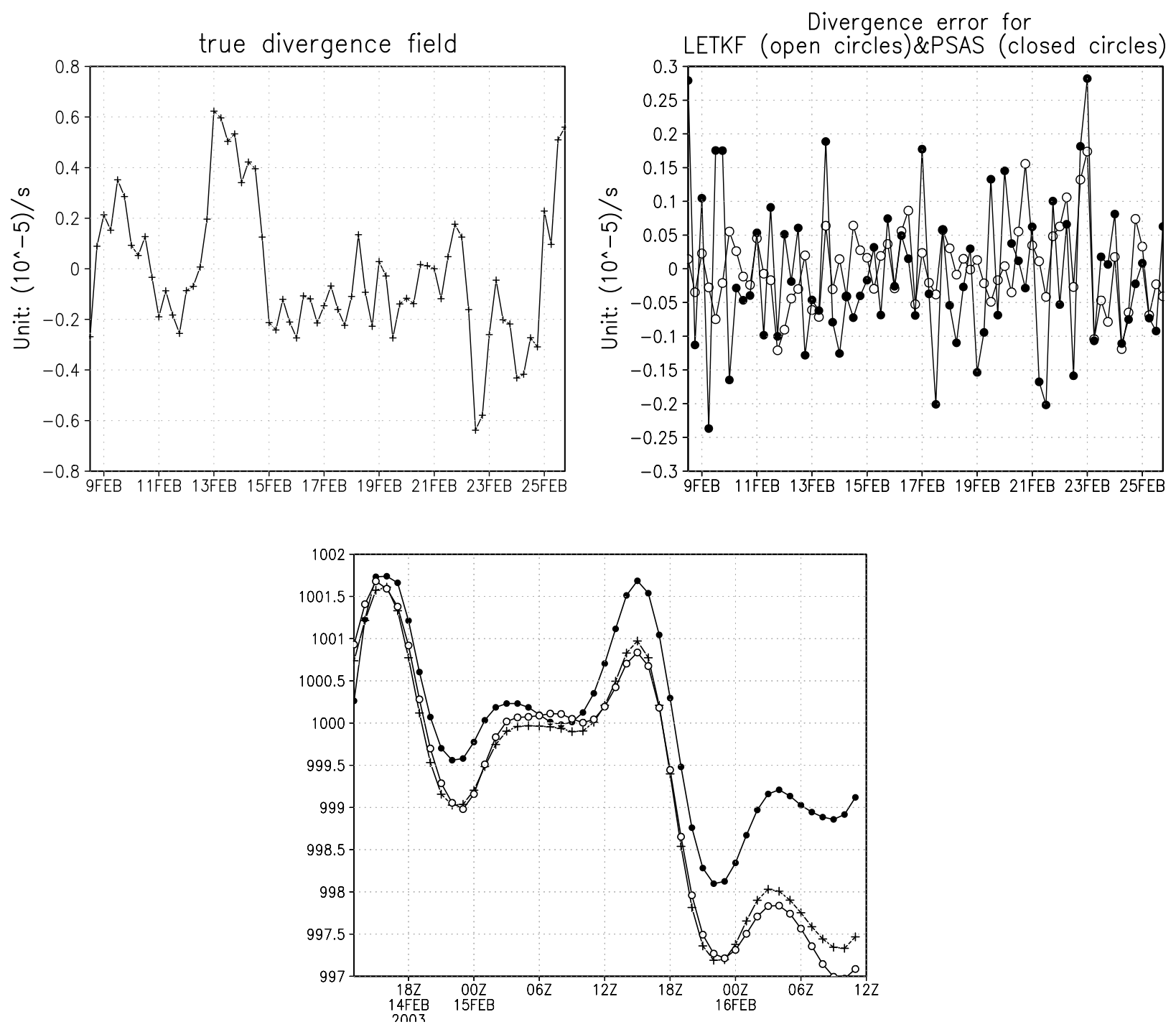

Fig. 7. Upper left panel: the "true" analyzed divergence field every $6 \mathrm{~h}$ at $32^{\circ} \mathrm{N}, 93^{\circ} \mathrm{W}$ on $700 \mathrm{hPa}$ (where there is a rawinsonde observation). Upper right panel: the divergence error for the LETKF (open circles) and PSAS (closed circles). The bottom panel: 2-day surface pressure forecast from 12:00 Z, 14 February at $32^{\circ} \mathrm{N}, 93^{\circ} \mathrm{W}$ (crosses show the true pressure, open circles are the LETKF forecast, and full circles are the PSAS forecast). The output interval is every hour.

\section{The relationship between analysis increments and background error}

The analysis increments (the difference between analysis state and background state) reflect the correction made to the background with the observed information. They are determined by the background error covariance, observation error, and observation innovation (the difference between observation and the background mean state in the observation space), as shown in Eq. (1) for PSAS and Eq. (8) for LETKF. The background error is the difference between background state and the truth, so that the optimal analysis increments should be equal and opposite sign of the background errors. We analyze reasons for the difference in the performance of the LETKF and PSAS by examining the relationship between analysis increment and background error on 12:00 UTC 12 February for both PSAS and the LETKF. This time is when the largest RMS error difference between LETKF and PSAS occurs (Fig. 2). The largest difference between these two schemes is observed over the ocean in the Southern Hemisphere (SH), especially between $30^{\circ} \mathrm{S}$ and $80^{\circ} \mathrm{S}, 120^{\circ} \mathrm{E}$ and $160^{\circ} \mathrm{W}$ where there is a deep trough associated with major weather development. Figure 8 shows the analysis increments (contours) and the background errors (colors) in the region described above. Both schemes extract useful information from the sparse observations, as indicated by the fact that the analysis increments generally have opposite sign to the background errors. However, in the LETKF (left panel in Fig. 8), the analysis increments line up with the background error generally better than that in PSAS, even in the areas 

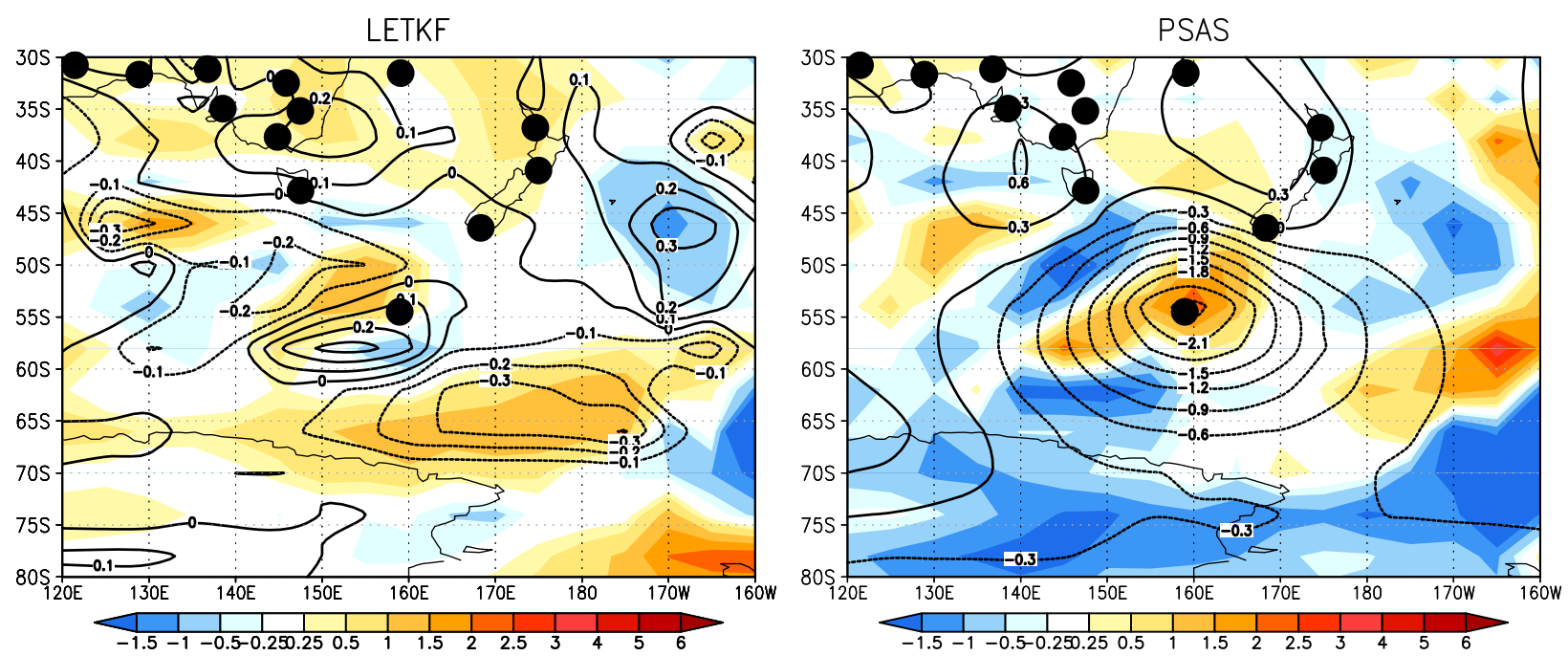

Fig. 8. $500 \mathrm{hPa}$ temperature analysis increments (contours) and background error (shaded) for the LETKF (left panel) and PSAS (right panel) at 12:00 Z, 12 February. The dots represent the rawinsonde observation locations.

without observations. Since the LETKF and PSAS assimilate the same observation and use the same observation error statistics, this different characteristic of analysis increments is mainly due to different background error statistics. The background error covariance estimated from the ensemble is able to extrapolate observation information to data sparse regions by accurately reflecting the shape of the errors of the day, so the analysis increments have the similar shape with the background error. Because PSAS has a constant isotropic background error covariance, it cannot estimate abrupt error changes in the shape and amplitude of background error. As a result, the structure of the PSAS temperature increments is significantly different from that of the background error (right panel in Fig. 8). In PSAS, the large analysis increments are observed around the observation locations, not in regions with large background error.

\section{Estimation of the sufficiency of the number of ensem- ble members used in the LETKF}

One measure of the sufficiency of the number of ensemble members is the consistency between the background uncertainty estimated from the background ensemble forecasts and the true background error. Ideally, with perfect model experiments and with enough ensemble members, the estimated background uncertainty should be same with the actual background error. When there are too few ensemble members, the background uncertainties estimated from the ensemble would be far from the actual background error.

Ensemble spread is used to represent the background uncertainty estimated from ensemble forecasts, which is the diagonal value of the background error covariance. We examine the ability of 40 ensemble members to adequately repre- sent the true uncertainty by comparing the ensemble spread to the actual ensemble mean error. Both quantities are averaged over the second month of the assimilation cycle. The time average of the ensemble spread is calculated as follows:

$S=\left[\frac{1}{T} \sum_{t=1}^{t=T} \frac{1}{k-1} \sum_{i=1}^{i=k}\left(\boldsymbol{x}_{i}^{b}-\overline{\boldsymbol{x}}^{b}\right)^{2}\right]^{\frac{1}{2}}$,

Here $S$ represents the time-averaged ensemble spread of any dynamical variable at any grid point, where $\boldsymbol{x}_{i}^{b}$ is the $\mathrm{i}^{\text {th }}$ background ensemble member and $\overline{\boldsymbol{x}}^{b}$ is the background mean state at that grid point. The error of the ensemble mean is measured by the distance between the background ensemble mean and the true state:

$V=\left[\frac{1}{T} \sum_{i=1}^{i=T}\left(\overline{\boldsymbol{x}}^{b}-\boldsymbol{x}^{t}\right)^{2}\right]^{\frac{1}{2}}$,

where $x^{t}$ is the true state at that grid point. If the data assimilation is optimal, and there are enough ensemble members to estimate the background error covariance, the background ensemble spread should be same as the error of the ensemble mean.

Figure 9 shows that the 40-member ensemble accurately estimates the shape of the background error. The centers of ensemble spread (contour) and ensemble mean error (shaded) are approximately at the same locations, and both fields have similar shapes. We examine the relative amplitude of the ensemble spread and ensemble mean error by calculating their ratio (which ideally should be equal to one). Overall, the 40-member ensemble accurately estimates the magnitudes of background uncertainty. The ratio of ensemble spread to ensemble-mean error is close to one in data dense regions, such as over land (Fig. 10). This suggests 


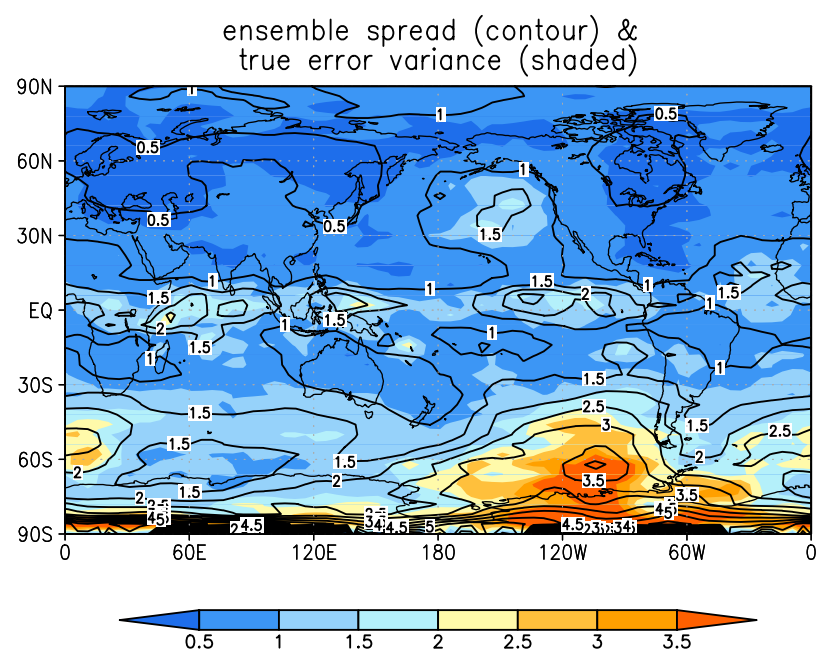

Fig. 9. Time average ensemble spread of zonal wind (averaged over February, contour; Unit: $\mathrm{m} / \mathrm{s}$ ) and the ensemble mean error (shades; Unit: $\mathrm{m} / \mathrm{s}$ ) at $500 \mathrm{hPa}$.

that larger inflation factors are required in the data dense region to keep a reasonable ensemble spread. In data sparse regions such as the tropics, the ratio of ensemble spread to variance is about 1.5-2 (Fig. 10), which suggests that the ensemble spread overestimates the background uncertainty, causing the analysis to give more weight to the observations than it should. Further tuning the inflation factor based on the observation coverage may improve the assimilation accuracy, since inflation should be different over data dense and data sparse regions.

Our results suggest that 40 ensemble members are enough to adequately capture the background uncertainty under the perfect model scenario with the limited observing system used and model resolution. We recognize that the resolution of the model used for this study is much coarser than that of operational models. Accordingly, more than 40 ensemble members may be required to estimate the background error covariance for operational models. However, the number of ensemble members required is not unlimited. Miyoshi and Yamane (2007) show that 80 ensemble members are enough to get good performance with T159/L48 (corresponding to a grid of $480 \times 240 \times 48)$ AGCM and much denser observations.

\section{Summary and discussion}

In this study we compare the performance of the LETKF with the NASA PSAS analysis system (GEOS-4 data assimilation system) by assimilating simulated rawinsonde observations on a finite volume GCM with horizontal resolution of $4^{\circ}$ by $5^{\circ}$ and 55 vertical levels. With 40 ensemble members, the LETKF analyses show significantly less RMS error than the PSAS analyses. The largest improvement of the

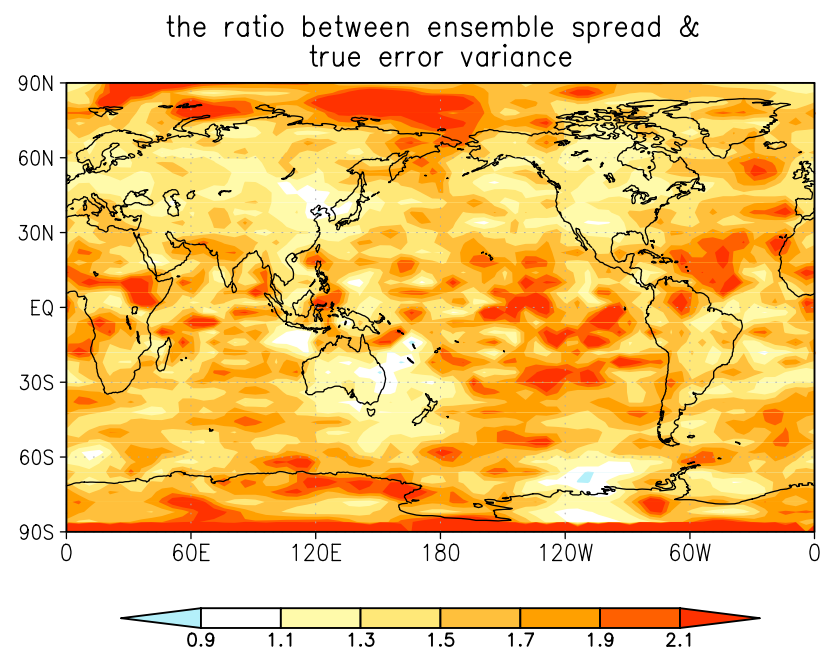

Fig. 10. The ratio of time average ensemble spread and ensemble mean error of zonal wind at $500 \mathrm{hPa}$.

LETKF over PSAS is found in regions with sparse observations, particularly in the Southern Hemisphere. This result is consistent with Whitaker et al. (2004, 2007) and Szunyogh et al. (2007) finding that ensemble Kalman filters have the most advantage over a 3D-Var in data sparse regions. The 5-day forecasts maintain this advantage. The forecast errors starting from the PSAS analysis in the $\mathrm{NH}$ slightly decrease in the first few hours before they start growing with time, indicating the presence of analysis imbalance that disperses as gravity waves during the initial geostrophic adjustment. By contrast, the initially smaller analysis error in the LETKF analysis grows exponentially, indicating better balance in the initial conditions.

We believe that the large improvement of the LETKF over the PSAS is due to the fact that the background error covariance used in the LETKF varies realistically with space and time, but the constant background error covariance used in the PSAS cannot reflect abrupt error changes in the background. As a result, the analysis increments structure are more similar (with opposite sign) to the background errors in the LETKF, whereas in PSAS the analysis corrections are more isotropic, and tend to be centered at observation locations. The accuracy of the background error covariance estimation is crucial to the performance of the LETKF scheme, and is related to the number of ensemble members. The agreement between ensemble spread and ensemble mean error suggests that forty ensemble members used in the LETKF are sufficient to capture most of the uncertainty in the global fvGCM forecast. Nevertheless, more ensemble members may be required in a higher resolution model.

Though the LETKF requires much more computational resources compared to PSAS, given enough processors, the actual wall-clock time can be comparable due to the highly parallel characteristics of the LETKF. The parallel computation characteristic comes from the localization of the LETKF 
scheme. In this version of the LETKF, the localization is based on the number of grid points in each local box around the center point. Only those observations within a local box are used to update the center grid point. Alternatively, the localization can be based on choosing the observations within a distance to update the center grid point, rather than using a local box (Hunt et al., 2007).

Although in this study we compared the LETKF with the 3D-Var analysis scheme used in the NASA GEOS-4 operational system, some caveats about the results should be mentioned. Our experiments are based on a perfect model scenario, in which we have avoided additional challenges associated with the presence of unknown observation and model errors. Also, the observational network only includes rawinsondes, which is much sparser than the operational observation network. Previous research shows that EnKF has more advantage in data sparse region (Whitaker et al., 2004) so that the advantages of the LETKF should be smaller for currently available operational observations than our results indicate. In addition, the error statistics of PSAS has not been well tuned, and the model resolution used is lower than that currently used in operations. Therefore our very encouraging results could be interpreted as an upper bound for the potential operational advantage of EnKF over 3D-Var. GEOS-4 has been replaced by the GEOS-5 system, which in the future should be also compared with the LETKF.

Acknowledgements. This work is part of the research towards the fulfillment of the doctorate requirement of the three leading authors, who have contributed equally. This research was partially supported by NSF ATM9328402, NASA NNG04GK29G and NNG04GK78A. We are grateful to Dirceu Herdies, Arlindo DaSilva and Shian-Jiann Lin for very helpful discussions. Two anonymous reviewers provided us with very constructive and detailed comments that helped clarify several aspects of this study.

Edited by: Z. Toth

Reviewed by: two anonymous referees

\section{References}

Anderson, J. L.: An ensemble adjustment Kalman filter for data assimilation, Mon. Weather Rev., 129, 2884-2903, 2001.

Anderson, J. L. and Anderson, S. L.: A Monte Carlo implementation of the nonlinear filtering problem to produce ensemble assimilations and forecasts, Mon. Weather Rev., 127, 2741-2758, 1999.

Bishop, C. H., Etherton, B., and Majumdar, S. J.: Adaptive sampling with the ensemble transform Kalman filter - Part I: Theoretical aspects, Mon. Weather Rev., 129, 420-436, 2001.

Bloom, S. C., Takacs, L. L., DaSilva, A. M., and Ledvina, D.: Data assimilation using incremental analysis updates, Mon. Weather Rev., 124, 1256-1271,1996.

Bloom, S. C., DaSilva, A., Dee, D., et al.: Documentation and validation of the Goddard Earth Observing System (GOES) Data Assimilation System - Version 4, Technical report series on global modeling and data assimilation, 26, available at: http: //gmao.gsfc.nasa.gov/systems/geos4/Bloom.pdf, 2005.

Cohn, S. E., DaSilva, A. M., Guo, J., Sienkiewicz, M., and Lamich, D.: Assessing the Effects of Data Selection with the DAO Physical-Space Statistical Analysis System, Mon. Weather Rev., 126, 2913-2926, 1998.

Courtier, P., Anderson, E., Heckley, W., Pailleux, J., Vasiljevic, D., Hamrud, M., Hollingsworth, A., Rabier, F., and Fisher, M.: The ECMWF implementation of three-dimensional variational assimilation (3D-Var) - I: Formulation, Q. J. Roy. Meteor. Soc., 124, 1783-1807, 1998.

Daley, R.: Atmospheric data analysis, Cambridge University Press, Cambridge, UK, 471 pp., 1991.

Dee, D. P. and DaSilva, A. M.: The choice of variable for atmospheric moisture analysis, Mon. Weather Rev., 131, 155-171, 2003.

Evensen, G.: Sequential data assimilation with a nonlinear quasigeostrophic model using Monte Carlo methods to forecast error statistics, J. Geophys. Res., 99(C5), 10 143-10 162, 1994.

Fertig, E. J., Hunt, B. R., Ott, E., and Szunyogh, I.: Assimilating nonlocal observations with a Local Ensemble Kalman Filter, Tellus A, 59, 719-730, 2007.

Hamill, T. M., Whitaker, J. S., and Snyder, C.: Distance-dependent filtering of background error covariance estimates in an ensemble Kalman filter, Mon. Weather Rev., 129, 2776-2790, 2001.

Harlim, J., Hunt, B. R., Kalnay, E., and Yorke, J. A.: Convex error growth, Phys. Rev. Lett., 94, 228501, doi:10.1103/PhysRevLett.94.228501, 2005.

Houtekamer, P. L. and Mitchell, H. L.: A sequential Ensemble Kalman Filter for atmospheric data assimilation, Mon. Weather Rev., 129, 123-137, 2001.

Houtekamer, P. L, Mitchell, H. L., Pellerin, G., Buehner, M., Charron, M., Spacek, L., and Hansen, B.: Atmospheric data assimilation with the ensemble Kalman filter: Results with real observations, Mon. Weather Rev., 133, 604-620, 2005.

Hunt, B. R., Kalnay, E., Kostelich, E. J., Ott, E., Patil, D. J., Sauer, T., Szunyogh, I., Yorke, J. A., and Zimin, A. V.: Fourdimensional ensemble Kalman filtering, Tellus A, 56(4), 273277, 2004.

Hunt, B. R., Kostelich, E. J., and Szunyogh, I.: Efficient Data Assimilation for Spatiotemporal Chaos: a Local Ensemble Transform Kalman Filter, Physica D, 230, 112-126, 2007.

Kalnay, E.: Atmospheric modeling, data assimilation and predictability, Cambridge Univ. Press, Cambridge, UK, 341 pp., 2003.

Kalnay, E., Li, H., Miyoshi, T., Yang, S.-C., and Ballabrera-Poy, J. B.: 4D-Var or ensemble Kalman filter?, Tellus A, 59, 758-773, 2007.

Kuhl, D. D., Szunyogh, I., Kostelich, E. J., Patil, D. J., Gyarmati, G., Oczkowski, M., Hunt, B. R., Kalnay, E., Ott, E., and Yorke, J. A.: Assessing predictability with a Local Ensemble Kalman Filter, J. Atmos. Sci., 64, 1116-1140, 2007.

Lin, S. J.: A "Vertically Lagrangian" Finite-Volume Dynamical Core for Global Models, Mon. Weather Rev., 132, 2293-2307, 2004.

Liu, J.: Applications of the LETKF to adaptive observations, analysis sensitivity, observation impact and assimilation of moisture, Ph. D thesis, University of Maryland, MD, USA, 2007.

Lynch, P. and Huang, X.-Y.: Initialization of the HIRLAM model 
using a digitial filter, Mon. Weather Rev., 120, 1019-1034, 1992.

Miyoshi, T. and Yamane, S.: Local Ensemble Transform Kalman Filtering with an AGCM at a T159/L48 resolution, Mon. Weather Rev., 135, 3841-3861, 2007.

Ott, E., Hunt, B. R., Szunyogh, I., Zimin, A. V., Kostelich, E. J., Corazza, M., Kalnay, E., Patil, D. J., and Yorke, J. A.: A Local Ensemble Kalman Filter for Atmospheric Data Assimilation, Tellus A, 56, 415-428, 2004.

Parrish, D. F. and Derber, J. C.: The National Meteorological Center's spectral statistical-interpolation analysis system, Mon. Weather Rev., 120, 1747-1763, 1992.

Szunyogh, I., Kostelich, E. J., Gyarmati, G., Kalnay, E., Hunt, B. R., Ott, E., Satterfield, E., and Yorke, J. A.: A Local Ensemble Transform Kalman filter data assimilation for the NCEP global model, Tellus A, 60, 113-130, 2008.

Szunyogh, I., Kostelich, E. J., Gyarmati, G., Patil, D. J., Hunt, B. R., Kalnay, E., Ott, E., and Yorke, J. A.: Assessing a Local Ensemble Kalman Filter: Perfect Model Experiments with the NCEP Global Model, Tellus A, 57, 528-545, 2005.
Tippett, M. K., Anderson, J. L., Bishop, C. H., Hamill, T. M., and Whitaker, J. S.: Ensemble square root filters, Mon. Weather Rev., 131, 1485-1490, 2003.

Toth, Z. and Kalnay, E.: Ensemble Forecasting at NCEP and the Breeding Method, Mon. Weather Rev., 125, 3297-3319, 1997.

Whitaker, J. S. and Hamill, T. M.: Ensemble data assimilation without perturbed observations, Mon. Weather Rev., 130, 1913-1924, 2002.

Whitaker, J. S., Compo, G. P., Wei, X. and Hamill, T. M.: Reanalysis without radiosondes using ensemble data assimilation, Mon. Weather Rev., 132, 1190-1200, 2004.

Whitaker, J. S., Hamill, T. M., Wei, X., Song, Y. C., and Toth, Z.: Ensemble data assimilation with NCEP global forecast system, Mon. Weather Rev., 136, 463-482, 2007. 